\title{
Transplante de microbiota fecal no tratamento da síndrome do intestino irritável: uma revisão sistemática
}

\author{
Fecal microbiota transplant in the treatment of irritable bowel syndrome: a systematic review \\ Rafaella De Nadai ${ }^{1}$, Carolina de Melo ${ }^{1}$, Jéssica Peroni Custódio ${ }^{1}$, Felipe Bertollo Ferreira ${ }^{2}$, Ana Paula Hamer \\ Sousa Clara ${ }^{2}$, Livia Zardo Trindade ${ }^{2}$, Fabiano Quarto Martins ${ }^{3}$, Izabelle Venturini Signorelli ${ }^{2}$
}

\section{Resumo}

Introdução: A Síndrome do Intestino Irritável (SII) é uma condição de alta prevalência e com um efeito significativo na qualidade de vida da população. É de etiologia multifatorial, incluindo alterações no eixo cérebro-intestino e na motilidade intestinal, porém, ainda não foi completamente elucidada. Dados recentes apontam sobre uma relação importante da disbiose intestinal na patogênese da SII. O transplante de microbiota fecal (TMF) tem a condição de reestabelecer a homeostase intestinal. Objetivos: Este trabalho objetiva a revisão de publicações científicas que associam o uso do TMF no tratamento da SII, para a posterior análise de sua eficácia terapêutica. Metodologia: A busca literária foi realizada no acervo MEDLINE, através do PubMed, onde angariamos 120 artigos. Através de critérios de inclusão e exclusão sobraram 9 publicações que foram analisados. Resultados: A partir da analise dos estudos, um total de 91 pacientes foram avaliados. O tratamento revelou melhora sintomática em $85.71 \%$ dos casos. As diferenças ente os 9 estudos inclusos devem ser levadas em consideração. Não houve homogeneidade entre modelos, vias e uso do antibiótico pré-TMF. Conclusão: O TMF se mostrou eficaz no tratamento da SII, porém são necessários estudos clínicos com maior impacto científico para concretizar esta nova modalidade terapêutica.

Descritores: Síndrome do intestino irritável, Transplante de microbiota fecal, Microbioma gastrointestinal

1. Acadêmica em Medicina da Escola Superior de Ciências da Santa Casa de Misericórdia de Vitória (EMESCAM)

2. Professor da Cadeira de Gastroenterologia e Semiologia da Escola Superior de Ciências da Santa Casa de Misericórdia de Vitória (EMESCAM)

3. Professor da Cadeira de Gastroenterologia e Clínica Médica da Escola Superior de Ciências da Santa Casa de Misericórdia de Vitória (EMESCAM)

Trabalho realizado: Escola Superior de Ciências da Santa Casa de Misericórdia de Vitória (EMESCAM)

Endereço para correspondência:. Rafaella De Nadai. Rua Desembargador Euripedes Queiros do Valle, 91, Jardim Camburi -29090-290 - Vitória-ES. E-mail: denadairafaella@gmail.com

\begin{abstract}
Background: The Irritable Bowel Syndrome (IBS) is a condition of high prevalence and has a significant effect when it comes to quality of life. It is a Functional Gastrointestinal disorder (FGID) of multifactorial etiology, including alterations in the gut-brain axis and intestinal motility, although, it is not yet fully comprehended. Recent data points to an important intestinal disbiosis as part of the pathogenesis of IBS. The Fecal Microbiota Transplant (FMT) has already been established as an alternative to the treatment of Clostridium difficile infection, condition that is also related to alterations of intestinal microbiota. Therefore, FMT is now being studied with the same goal, normalizing intestinal homeostasis in patients with IBS. Aim: To review scientific articles that associate the use of FMT as treatment of IBS, for further analysis of its therapeutic efficiency. Methods: The literary search was made at MEDLINE, through PubMed, where we recover 120 papers. Among them, we analyzed only 9 articles, selected by inclusion and exclusion criteria previously established. Results: In the final analysis, a total of 91 patients were assessed. The treatment revealed an improvement of $85.71 \%$ of cases. The differences between the 9 included studies must be taken into consideration. Conclusion: The FMT has proven to be effective as treatment of IBS, however, more complex studies are needed to establish this new therapy.
\end{abstract}

Keywords: Irritable bowel syndrome, Fecal microbiota transplantation, Gastrointestinal microbiome

\section{Introdução}

O grupo dos Distúrbios Funcionais Gastrointestinais (DFGI) é composto por doenças crônicas, de etiologia multifatorial ${ }^{(1)}$ e de grande importância para a saúde pública ${ }^{(1-2)}$. A Síndrome do Intestino Irritável (SII) é a doença característica desse grupo e é prevalente em toda a população mundial, causando profundo efeito na qualidade de vida e gastos publicos com saúde ${ }^{(2)}$. Apesar disso, a fisiopatologia ainda não está completamente clara. Vários mecanismos como a dismotilidade gastrointestinal e a 
hipersensibilidade visceral tem sido mais comumente propostos para explicá-la. Além disso, as literaturas mais recentes sugerem a participação da interação entre o sistema imune da mucosa, barreira epitelial e fatores luminais, como alimentos e bactérias intestinais na fisiopatogenia ${ }^{(3)}$.

São considerados sintomas clínicos característicos: a dor abdominal, as mudanças no número de evacuações e as mudanças na consistência das fezes ${ }^{(4)}$. Esta última alteração clínica é classificada de acordo com a escala de Bristol (EB) e é responsável por determinar em qual subgrupo da SII o paciente se encontra, sendo esses subgrupos:

- Constipação (SII- C) em que o sintoma predominante é a constipação (EB 1-2 em > 25\% das evacuações e $<6-7$ em $25 \%$ das evacuações);

- Diarreia (SII-D) em que o sintoma predominante é a diarreia (EB 1-2 em $>25 \%$ das evacuações e $<6-7$ em $25 \%$ das evacuações);

- Misto (SII-M) em que os sintomas são mistos.

Existe ainda uma subclassificação especial, a SII pós-infecciosa, referente aos sintomas desencadeados após infecção bacteriana ou viral aguda ${ }^{(2)}$.

Estas apresentações características da SII normalmente se associam a sintomas psicológicos. Estudos epidemiológicos estimam uma prevalência em torno de 40 a $90 \%$ transtornos de depressão e ansiedade nesses pacientes ${ }^{(5)}$.

O diagnóstico da SII é alcançado através dos dados clínicos, tomando por base os novos critérios de Roma $\mathrm{IV}^{(4)}$, o qual exige a presença de um critério obrigatório (dor abdominal frequente, pelo menos 1 vez por semana, mantida nos últimos 3 meses e recorrente há mais de 6 meses) associada a dois ou mais dos seguintes: relação com evacuação (piora ou melhora da dor), mudança nas frequencia das evacuações e alteração na EB. Para confirmação diagnóstica, devem-se realizar os exames orientados pela anamnese e caso haja presença de sinais de alarme, deve ser realizada colonoscopia para excluir causas orgânicas ${ }^{(6)}$.

A SII não possui tratamento definitivo, mas pode ser controlada a partir da eliminação de fatores exacerbantes e manejo dos sintomas ${ }^{(2)}$. Faz parte do tratamento não famacológico evitar alimentos que desencadeiem o início dos sintomas, consumir o mínimo de "fast food" possível e praticar exercícios físicos ${ }^{(6)}$.

Em relação ao tratamento farmacológico, ainda não há medicação padrão. O pilar para o manejo da dor abdominal é o uso dos antiespasmódicos. Os antidepressivos também possuem propriedades analgésicas, contudo, seu uso pode ser indicado por um curto período. O uso de probióticos como Bifidobacterium sp. e Saccharomyces boulardii também demonstraram eficácia na $\mathrm{SII}^{(7)}$. Caso a tentativa medicamentosa não obtiver resposta por 3 meses, deve ser encerrada ${ }^{(1)}$.

\section{Associação entre microbiota intestinal e SII}

As alterações na microbiota intestinal têm sido diretamente relacionadas com imunidade, motilidade e alterações neurológicas características da SII ${ }^{(8)}$. A comunicação entre o intestino e o sistema nervoso central é bidirecional e, em parte, também é modulada pela microbiota intestinal ${ }^{(9)}$. Outros estudos admitem que a SII pode ser precedida por estressores que afetam a microbiota e através da alteração da sensibilidade visceral e alterações de inervação autonômica, diminuem o limiar de dor e alteram o fluxo sanguíneo na mucosa retal, características observadas na $\mathrm{SII}^{(8)}$.

Estudos vêm pontuando diferenças constitucionais entre os pacientes com SII e seus respectivos controles. Nos pacientes com a síndrome, ocorre diminuição de Lactobacillus e Bifidobacterium e um aumento de Enterobacteraceae spp quando comparado com os controles. Além disso, já foi concluído que existe diferença na composição da microbiota até mesmo dentre os subtipos de $\mathrm{SII}{ }^{(8)}$.

O exposto acima sugere que a restauração da homeostase intestinal através do transplante de microbiota fecal (TMF) pode levar a melhora sintomática ${ }^{(1)}$.

\section{Considerações sobre o transplante fecal}

O TMF consiste na transferência da microbiota intestinal de um indivíduo para o outro, o que pode ser feito através da cápsulas orais, sonda nasogástrica ou nasoentérica, endoscopia digestiva alta (EDA), colonoscopia e enema.

A microbiota intestinal é adquirida pela coleta das fezes. Os pacientes em geral, têm uma aceitação boa em relação ao transplante. Porém, a administração de fezes realizada por via do trato gastrointestinal (TGI) superior é considerada uma opção mais desagradável pelos pacientes em comparação com a colonoscopia e enema ${ }^{(7)}$.

Os doadores e o respectivo material doado, devem ser criteriosamente rastreados a fim de diminuir o risco de transmissão de doenças. No entanto, essas diretrizes são baseadas na opinião de especialistas e não em evidências científicas ${ }^{(9)}$.

Atualmente, a segurança do TMF tem sido predominantemente estudada no tratameno da infecção do Clostridium difficile (ICD) (2). Em geral o TMF é considerado um tratamento seguro, com taxas míninas de efeitos adversos leves. No entanto, a segurança continua a ser a principal consideração e um número maior de observações em ensaios clínicos controlados é necessário. Além disso, como os efeitos colaterais em longo prazo da TMF não são conhecidos, a criação de uma base de dados nacional para monitorar eventos adversos deve ser considerada ${ }^{(1)}$. 
A SII tem uma alta prevalência na população e tem um efeito importante na qualidade de vida e nos custos de saúde. Visto isso, o TMF é uma alternativa de tratamento benéfico, uma vez que atua na patogênese da síndrome, e não nos sintomas subsequentes, como faz a terapia tradicional. Além disso, é uma estratégia com melhor custo/benefício em relação às drogas de uso contínuo.

\section{Discussão e Conclusões}

A etiologia da SII ainda não está completamente elucidada, no entanto se sabe que o ecossistema intestinal tem participação importante no desenvolvimento e manutenção da doença ${ }^{(9)}$. Nessa linha, o TMF abre uma possibilidade de manipulação da microbiota e melhora dos sintomas da SII. Esta revisão sistemática da literatura mostrou que, nos trabalhos publicados, o tratamento com transplante parece ser benéfico, sendo observada alguma melhora sintomática em 85,71\% dos pacientes.

Após busca incessante, encontrou-se 9 estudos ${ }^{(10-18)}$ sobre o tema descrito. Esses artigos configuram toda a literatura disponível na plataforma do MEDLINE. Apesar da metodologia de inclusão ter sido variável, 91 pacientes ao todo foram expostos ao tratamento com TMF nos estudos analisados. Em todos estes houve melhoras sintomáticas pós-transplante nas suas respectivas populações.

Além destas vantagens nos sintomas diretamente vinculados à síndrome, os pacientes que realizaram o TMF tiveram melhora no status psicológico, segundo o estudo de Mizuno et al, $2017^{(16)}$ que utilizou a escala de Hamilton Rating.

Nos artigos lidos, observou-se ampla variação de métodos para a análise da evolução dos pacientes após o TMF, o que dificulta a formulação de um conceito que englobe todos os trabalhos a respeito da melhora sintomática. Além disso, não há nenhuma explicação para a frequência com que o TMF foi realizado nem para o volume de fezes necessário para um bom resultado. Apenas o estudo de Li et al, $2017^{(13)}$ comparou, além das variáveis supracitadas, a via de administração, concluindo que não houve diferença significativa entre as três. Não obstante, somente esse trabalho não é suficiente para levar tal conclusão para a prática clinica, sendo necessários estudos posteriores com esse objetivo.

A ocorrência de eventos adversos (EA) graves relacionados ao TMF foi nula. Apenas dois trabalhos ${ }^{(13-14)}$ relataram EA, ao quais foram associados às vias utilizadas para a administração do TMF. Todos os eventos desapareceram com o fim do procedimento ou durante a observação imediata pós TMF. Esses achados conferem sentimento de segurança para o tratamento proposto.
Mizuno et al, 2017 $7^{(16)}$ foram os primeiros a levar em consideração a relação das fezes dos doadores com eficácia do TMF. Esse autor concluiu que a eficácia do tratamento está diretamente ligada à microbiota dos doadores, em especial, a presença de altos níveis do gênero Bifidobacterium sp. em suas fezes. Em contrapartida, o efeito terapêutico do transplante não tem relação com o aumento desse gênero na microbiota dos pacientes após o TMF. Isso indica que não é necessária a proliferação de bactérias do gênero Bifidobacterium no trato intestinal desses pacientes para induzir o aumento da diversidade de sua microbiota e, consequentemente, a melhora clínica desses pacientes. Logo, são necessários mais estudos a fim de elucidas o papel dessa bactéria no TGI.

Além do screening geral para doenças infecciosas e a utilização de pessoas saudáveis como doadoras, poucos são os critérios de inclusão para este grupo. Não foram constatadas diferenças significativas entre a idade e sexo de doadores e receptores. Além da presença de Bifidobacterium sp. nas fezes de doadores ser um possível preditor positivo para a eficácia do TMF, não se têm muitas informações sobre o grau de interferência que esses doadores têm sobre a melhora clínica de seus receptores, visto que apenas um estudo avaliou esta relação.

A falta de homogeneidade entre os estudos avaliados dificulta a análise dos resultados como um todo devido à ausência de um protocolo específico para a realização do procedimento do transplante. Além disso, existe um risco de viés de resultados positivos ao lidar com pequenos ensaios e relatos de casos nos quais os pesquisadores somente publicam casos em que a melhoria foi relatada. No entanto, os estudos incluídos nesta revisão estabelecem um trabalho de base para ensaios clínicos futuros com maior impacto científico, visto que o TMF se mostrou eficaz, mesmo que temporariamente, como terapêutica da SII.

\section{Referências}

1. Pinn DM, Aroniadis OC, Brandt LJ. Is fecal microbiota transplantation (FMT) an effective treatment for patients with functional gastrointestinal disorders (FGID)? Neurogastroenterol Motil. 2015; 27(1):19-29.

2. Soares LR. Irritable bowel syndrome: a clinical review. World J Gastroenterol. 2014; 20(34): 12144-60.

3. Camilleri M, Lasch K, Zhou W. Irritable bowel syndrome: methods, mechanisms, and pathophysiology. Am J Physiol Gastrointest Liver Physiol. 2012; 303(7):G775-85.

4. Grzesiak M, Beszłej JA, Waszczuk E, Szechiński M, SzewczukBogusławska M, Frydecka D, et al. Serotonin-related gene variants in patients with irritable bowel syndrome and depressive or anxiety disorders. Gastroenterol Res Pract. 2017; 2017: 4290430.

5. Schmulson MJ, Drossman DA. What is new in Rome IV. J Neurogastroenterol Motil. 2017; 23(2): 151-63.

6. American College of Gastroenterology Task Force on Irritable Bowel Syndrome, Brandt LJ, Chey WD, Foxx-Orenstein AE, 
Schiller LR, Schoenfeld PS, et al. An evidence-based position statement on the management of irritable bowel syndrome. Am J Gastroenterol. 2009;104 (Suppl 1):S1-35.

7. Brandt LJ, Aroniadis OC. An overview of fecal microbiota transplantation: techniques, indications, and outcomes. Gastrointest Endosc 2013; 78(2):240-9

8. Ford AC, Talley NJ, Schoenfeld PS, Quigley EM, Moayyedi P. Efficacy of antidepressants and psychological therapies in irritable bowel syndrome: systematic review and meta-analysis. Gut. 2009; 58(3):367-78.

9. Pinn D, Aroniadis O, Brandt L). Follow-up study of fecal microbiota transplantation (FMT) for the treatment of refractory irritable bowel syndrome (IBS. Am J Gastroenterol. 2013; 108(suppl.1):S563.

10. DuPont AW, Jiang ZD, Harold SA, Snyder N, Galler GW, Garcia-Torres F, et al. Motility abnormalities in irritable bowel syndrome. Digestion. 2014; 89(2):119-23

11. Syzenko G, Budovska L, Puchkov K. P0397 Efficiency of FMT in cases of 'Treatment-resistant' IBS. United European Gastroenterol J. 4(5S):A294.

12. Cruz Aguilar R, Buch T, Bajbouj. Fecal microbiota transplantation as a novel therapy for irritable bowel syndrome with predominant diarrhea. Neurogastroenterol Motil. 2015;27(Suppl 2):110.

13. Li N, Tian H, Ma C, Ding C, Ge X, Gu L, et al. [Efficacy analysis of fecal microbiota transplantation in the treatment of 406 cases with gastrointestinal disorders]. Zhonghua Wei Chang Wai Ke Za Zhi. 2017; 20(1):40-6. Article in Chinese.
14. Shin Y, Hong J, Bang B, Kim H, Kwon K. OP treatment of irritable bowel syndrome with fecal microbiota transplantation: a case series of 10 patients. United European Gastroenterol J. 2016; 2 (Suppl 1):p257.

15. Mazzawi T, Lied G, El-Salhy M, Gilja O, Hatlebakk J, Hausken T. Effect of faecal microbiota transplantation on symptoms and duodenal enteroendocrine cells in patients with irritable bowel syndrome. United European Gastroenterol J. 2016; (Suppl 1):p1818.

16. Mizuno S, Masaoka T, Naganuma M, Kishimoto T, Kitazawa M, Kurokawa S, et al. Bifidobacterium-rich fecal donor may be a positive predictor for successful fecal microbiota transplantation in patients with irritable bowel syndrome. Digestion. 2017; 96(1):29-38

17. Holvoet T, Boelens J, Joossens M, Raes J, Vos MD, Looze DD. Tu2025 Fecal microbiota transplantation in irritable bowel syndrome with bloating: results from a prospective pilot study. Gastroenterology. 2015; 148(4 suppl. 1):S963-S964.

18. Andrews PJ, Barnes P, Borody TJ. Chronic constipation reversed by restoration of bowel flora - a case and a hypothesis. Eur J Gastroenterol Hepatol. 1992; 4(3):245-7.

Artigo recebido: $23 / 10 / 2017$

Artigo aprovado: 14/11/2017 